\title{
Cascade médicamenteuse chez la personne âgée
}

\author{
Shelley A. Sternberg MD, Shiri Guy-Alfandary DPharm, Paula A. Rochon MD
}

Citation : CMAJ 2021 February 8;193:E215. doi : 10.1503/cmaj.201564-f

Voir la version anglaise de l’article ici : www.cmaj.ca/lookup/doi/10.1503/cmaj.201564

\section{1}

La cascade médicamenteuse fait référence à une séquence

d'événements au cours de laquelle l'effet secondaire d'un médicament est interprété à tort comme un nouveau problème de santé, conduisant à l'ajout d'un autre médicament potentiellement évitable ${ }^{1}$

Les anticalciques, par exemple, peuvent entraîner un œdème des chevilles pour lequel un diurétique serait prescrit. Dans une étude de cohorte, cette cascade en particulier a été observée chez 9,5\% des personnes âgées à qui on venait de prescrire un anticalcique (annexe 1 , accessible en anglais au www.cmaj.ca/lookup/doi/10.1503/cmaj.201564/tab-related-content)2.

2 Des effets secondaires graves peuvent entraîner l'hospitalisation Dans un cas, un patient a présenté de la toux après avoir commencé à prendre un inhibiteur de l'enzyme de conversion de l'angiotensine. On lui a prescrit un sirop antitussif renfermant de la guaïfénésine et de la codéine qui a provoqué de la léthargie ${ }^{3}$. On a ensuite débuté de la lévofloxacine pour une pneumonie présumée; il s'en est suivi de la diarrhée, puis du délire, qui ont abouti à une hospitalisation.

La cascade médicamenteuse contribue au phénomène

de polypharmacie inappropriée, particulièrement chez la personne âgée

Les patients atteints de maladies chroniques qui présentent des syndromes gériatriques nécessitant des schémas thérapeutiques complexes sont plus à risque à l'égard de la polypharmacie inappropriée, voire problématique ${ }^{4}$.

4.

Il est possible d'identifier les cascades médicamenteuses et de prévenir la polypharmacie inappropriée ${ }^{1}$

Les prescripteurs devraient se demander : « le symptôme du patient pourrait-il être l'effet secondaire d'un médicament? » et « un nouveau médicament est-il envisagé pour traiter l'effet secondaire possible d'un traitement médicamenteux en cours? » Si la réponse à l'une ou l'autre de ces questions est oui, les prescripteurs devraient alors se demander : « peut-on changer le médicament en cause pour un agent plus sécuritaire ou peut-on en réduire la dose et éliminer ainsi le recours au médicament subséquent? » et « le patient a-t-il besoin du médicament initial en premier lieu ou peut-on le cesser? ».

\section{Envisager la déprescription lorsqu'on identifie une cascade médicamenteuse}

Déprescrire consiste à réduire progressivement ou à cesser les médicaments qui ne sont pas indiqués en fonction des priorités des patients afin de réduire la polypharmacie et d'améliorer les résultats chez les patients 5 .

\section{Références}

1. Rochon PA, Gurwitz JH. The prescribing cascade revisited. Lancet 2017;389:1778-80.

2. Savage RD, Visentin JD, Bronskill SE, et al. Evaluation of a common prescribing cascade of calcium channel blockers and diuretics in older adults with hypertension. JAMA Intern Med 2020;180:643-51.

3. Liu PT, Argento VS, Skudlarska BA. Prescribing cascade in an 80-year-old Japanese immigrant. Geriatr Gerontol Int 2009;9:402-4.

4. Duerden M, Avery T, Payne R. Polypharmacy and medicines optimization: making it safe and sound. London: The King's Fund; 2013. Accessible ici : www.kingsfund.org.uk/ sites/files/kf/field/field_publication_file/polypharmacy -and-medicines-optimisation-kingsfund-nov13.pdf (consulté le 16 juin 2020).

5. Scott IA, Hilmer SN, Reeve E, et al. Reducing inappropriate polypharmacy: the process of deprescribing. JAMA Intern Med 2015;175:827-34.

Intérêts concurrents : Aucun déclaré.

Cet article a été révisé par des pairs.

Affiliations : Service de santé Maccabi (Sternberg), Modi'in, Israël; Service de santé Maccabi (GuyAlfandary), Tel-Aviv, Israël; Hôpital Women's College (Rochon), Université de Toronto, Toronto, Ont.

Propriété intellectuelle du contenu : Il s'agit d'un article en libre accès distribué conformément aux modalités de la licence Creative Commons Attribution (CC BY-NC-ND 4,0), qui permet l'utilisation, la diffusion et la reproduction dans tout médium à la condition que la publication originale soit adéquatement citée, que l'utilisation se fasse à des fins non commerciales (c.-à-d., recherche ou éducation) et qu'aucune modification ni adaptation n'y soit apportée. Voir : https://creativecommons.org/ licenses/by-nc-nd/4.0/deed.fr.

Financement : Le projet iKascade bénéficie de l'aide financière de GENDER NET Plus (GNP-1782) en partenariat avec les Instituts de recherche en santé du Canada (Institut de la santé des femmes et des hommes et Institut du vieillissement), le Conseil irlandais de la recherche (Irlande), le ministère de la Santé (Italie) et le ministère des Sciences, de la Technologie et de l'Espace (Israël).

Correspondance : Shelley Sternberg, sternb_sh@mac.org.il 